Témoigner Témoigner. Entre histoire et mémoire

Getuigen Revue pluridisciplinaire de la Fondation Auschwitz

119 | 2014

Il y a 70 ans, Auschwitz. Retour sur Primo Levi

\title{
Korngold à Musiques interdites
}

Korngold's Kathrin by Forbidden Music

Korngold op Musiques interdites

Jean-Luc Clairet

\section{(2) OpenEdition}

Journals

Édition électronique

URL : http://journals.openedition.org/temoigner/1606

DOI : $10.4000 /$ temoigner.1606

ISSN : 2506-6390

Éditeur :

Éditions du Centre d'études et de documentation Mémoire d'Auschwitz, Éditions Kimé

Édition imprimée

Date de publication : 31 décembre 2014

Pagination : $37-40$

ISBN : 978-2-84174-688-0

ISSN : 2031-4183

Référence électronique

Jean-Luc Clairet, "Korngold à Musiques interdites », Témoigner. Entre histoire et mémoire [En ligne], 119 |

2014, mis en ligne le 01 janvier 2016, consulté le 23 octobre 2020. URL : http://

journals.openedition.org/temoigner/1606 ; DOI : https://doi.org/10.4000/temoigner.1606

Tous droits réservés 


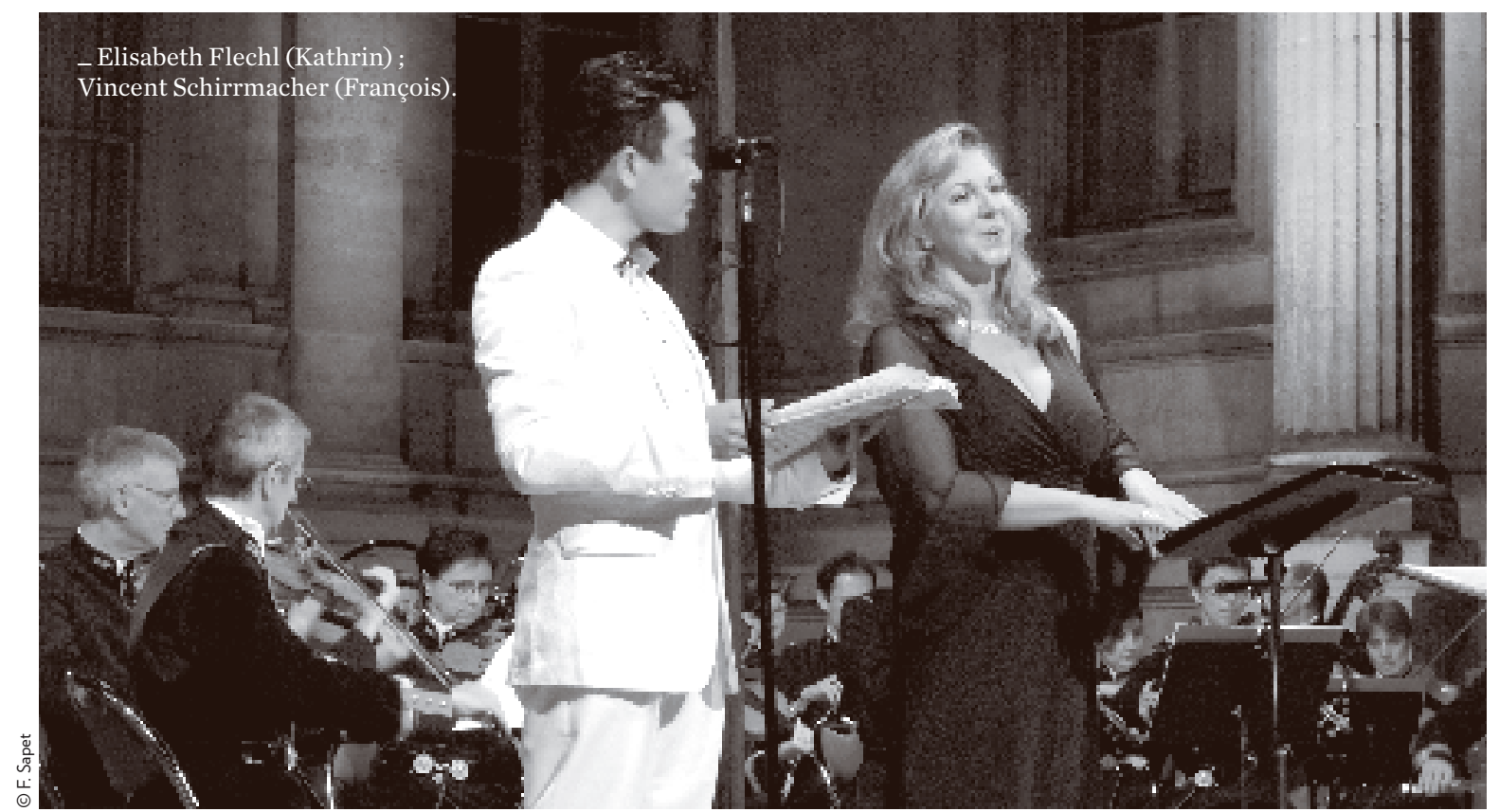

\section{KORNGOLD A MUSIQUES INTERDITES}

\section{MUSIQUE Le festival Musiques Interdites de Marseille permet de ressusciter quelques joyaux de la grande musique que la sombre histoire des répressions totalitaires a écartés de la scène. II en est ainsi de Die Kathrin de Korngold, qui aurait dû être montée en 1938 à Vienne, si la rage nazie ne l'avait rendue muette.}

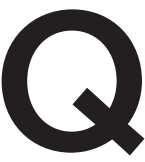

uoiqu'en puissent penser certains aujourd'hui, à une époque où des hommes et des femmes sont tentés d'offrir un peu de pouvoir à des gens pour qui la Culture est non seulement très secondaire, mais sous les prétextes les plus fallacieux, l'ennemi à abattre en priorité, il faut rappeler que le totalitarisme laisse toujours des traces. Il est très dur de reconstruire ce qui a été écarté. Les nazis l'ont démontré, eux qui, le plus spectaculairement, ont non seulement assassiné les talents les plus prometteurs (ainsi Pavel Haas dès son arrivée à Auschwitz en 1944, après que l'on eut exploité ses dons à Terezin), mais ont martelé le terme Entartete Musik (Musique dégénérée) au sujet d'une foultitude de compositeurs qui, pour la plupart, n'ont toujours pas retrouvé la place destinée à être la leur dans l'Histoire de la Musique. Emblématique de cette extermination physique autant que langagière fut Erich Wolfgang Korngold.

Korngold, élève de Zemlinsky (il aurait pu tomber plus mal!), admiré par Richard Strauss et Puccini euxmêmes (excusez du peu!), Korngold s'inscrit parfaitement dans la trajectoire des compositeurs du XX $\mathrm{X}^{\mathrm{e}}$ siècle et apporte sa réponse au courant romantique finissant confronté au sérialisme naissant. S’inscrivant dans la filiation des grands romantiques, davantage Femme •.॰ 
•. sans ombre que Pierrot lunaire, sa musique devient enfin audible en ce début de millénaire.

Mais quel parcours semé d'embûches ! Exécuté une première fois par le jugement nazi qui alla jusqu’à ordonner de brûler toutes ses partitions (fort heureusement, par l'entremise de son second éditeur, Weinberger, il n'en fut rien), Korngold le fut peut-être davantage encore par les jugements qui perdurèrent à son sujet jusqu’à il y a très peu de temps. On a encore parfaitement en mémoire les lazzi condescendants que colportèrent bien des articles de presse lorsque parut, en 1975, le splendide enregistrement RCA de Die tote Stadt (La Ville morte), l'opéra qui lui apporta la célébrité à 23 ans. Ce fut la même serial attitude, dans la France musicale bien-pensante de l'époque, à la sortie du très soigné (temps béni où un opéra était présenté dans un joli coffret avec livret au moins trilingue !) Violanta chez CBS en 1980. Difficile, dans ces conditions, d'écouter sereinement une musique que l'on nous disait boursouflée, avatar monstrueux des drames straussiens, qualifiée, suprême injure, de « musique de film! » Il faut dire que la musique de film n'inspirait pas le respect dont elle jouit aujourd'hui : bandes-son sophistiquées de plusieurs films de Resnais, partition ultra-contemporaine de Jerry Goldsmith pour le blockbuster La Planète des singes, mais à l'opposé reconnaissance unanime du mélodiste hors pair que fut Georges Delerue... Il en fallut du temps pour que les toits des chapelles musicales finissent par se lézarder enfin...

Korngold fut bien évidemment un précurseur en ce domaine où il excella à brouiller les genres. Fuyant la barbarie nazie, il s'installa définitivement en Amérique où il avait déjà fait ses preuves et fut un compositeur adulé par Hollywood, autre statut rédhibitoire. Il composa cinq opéras : Der Ring des Polykrates, Violanta (1916), Die tote Stadt (1920), Das Wunder der Heliane (1927), Die Kathrin (1937). Sa mort, en 1957, l'empêcha d'en achever un sixième. Au regard de l'exigence vocale qu'ils requièrent (des Siegfried, des Minnie, pas moins), les opéras de Korngold sont peu montés. Leur difficulté d'accès, due à l'opulence sophistiquée d'un discours musical plus Zemlinsky que Wagner, donc avare en mélodies prégnantes, à l'impact moins immédiat de leurs livrets, est, elle aussi, en cause. Néanmoins, les vestes se retournent peu à peu et chaque apparition de Die tote Stadt, son opéra le plus célèbre, soulève dorénavant enthousiasme et considération.

Cinquante ans après l'anathème nazi, au mitan des

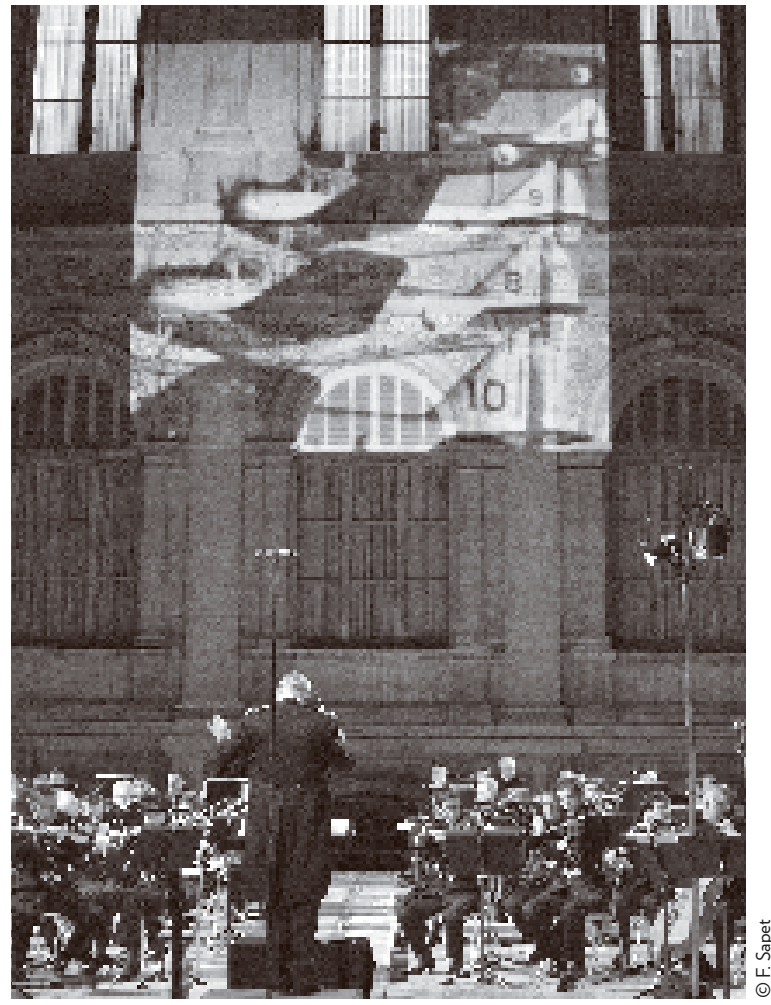

_ Orchestre symphonique de la Garde Républicaine, direction : Sébastien Billard.

années 1990, la firme Decca lança une nouvelle collection d'enregistrements audacieusement baptisée Entartete Musik. Elle offrit la splendeur légendaire de ses prises de son aux exclus magnifiques. Apparut alors foultitude de noms inconnus et de sons nouveaux : Sarlatan de Pavel Haas, Die Vögel de Brauenfels ou Die Gezeichneten de Schrecker voisinèrent alors dans les bacs avec La Walkyrie, Cosifan tutte ou même Les Troyens dont les auteurs avaient quant à eux déjà plus qu'imprimé leur marque dans l'Histoire la Musique. Collection magnifique, qui rendit caduque l'effroyable assertion du premier éditeur de Korngold : « Les Juifs n’ont pas de puissance créatrice! »

Le Festival Musiques Interdites de Marseille, en partenariat avec le Centre culturel autrichien à Paris et l'Opéra de Marseille, s'inscrit salutairement dans la même démarche. Profitant de l'érudition sans faille de Michel Pastore (entre autres producteur, programmateur, metteur en scène, librettiste...), il compte dans son 
comité d'honneur les noms prestigieux de Laure Adler, Elie Wiesel, Renée Auphan ou encore feu Stéphane Hessel. Robert Badinter est l'invité d'honneur de cette $9^{\text {e }}$ édition. L’idée est de pérenniser un « Cérémonial de la Mémoire » en réinvestissant les lieux mêmes qui ont vu la tentative d'anéantissement de celle-ci (en 2007, le Festival se produisit conjointement à Marseille et à Terezin). Conférences, films, récitals, lectures, ciné-concerts, opéras s’invitent à Marseille, ville emblématique s'il en est : Capitale de l'exil durant laSeconde Guerre mondiale, elle se veut «Symbole des réconciliations ». La Capitale européenne de la Culture qu'elle est devenue a en toute logique labellisé en 2013 cet incontournable Festival Musiques Interdites.

En 2014, pour sa $9^{\mathrm{e}}$ édition prévue sur 4 jours, le Festival Musiques Interdites aura vu son déroulement bousculé par le conflit des intermittents du spectacle. Ont été reportés au week-end du 13 septembre, la création de Philippe Hersant couplée avec Pierrot lunaire ainsi que le film-concert Meyrowitz's Marseilles Barriers, le ciné-concert La ville sans juifs. Seule rescapée, ce 8 juillet, la création française de Die Kathrin, ultime opus lyrique de Korngold.

S'inscrivant dans la Charte du Festival, le choix de la Préfecture de Marseille s'avère très judicieux pour cet opéra hanté par les thèmes de la frontière et de l'exil : c'est effectivement dans cette même cour où sont installées ce soir quatre centaines de chaises, que défilèrent jadis les candidats à l'exil lors de la période la plus sombre de notre Histoire. En ce 8 juillet 2014, à l'étage, ce sont les négociations avec la CSNM qui se prolongent tard dans la nuit et qui remplacent les démarches administratives de jadis. En contrebas, L’Orchestre Symphonique de la Garde républicaine et sept solistes nous font entendre Die Kathrin, opéra que son auteur mit cinq ans à composer.

La création de Die Kathrin, prévue en 1938 à Vienne, fut annulée in extremis sur ordre des nazis et, hormis un enregistrement audio réalisé par la BBC en 1998 avec Melanie Diener dans le rôle-titre, cet opéra resté inédit en France, trouve enfin une manière de cadre idéal dans le Festival Musiques Interdites. Cadre néanmoins un peu rogné sur les bords par la version qu'en a voulu Michel Pastore pour cette création française. Sa Kathrin subit un drastique raccourcissement : ses $2 \mathrm{~h}$
50 (Die tote Stadt ne dépasse pas les 2 h 20) se voient ramenées à $1 \mathrm{~h} 40$ ! Les chœurs sont évacués. C'est une version de concert mise en espace de façon très sobre, avec quelques rares accessoires (un revolver, une étoile jaune, des brassards de milicien façon Lacombe Lucien) et rehaussée de quelques images (photos, vidéo, effets de lumières, titres des scènes et sur-titres défilent sur la façade du bâtiment). De fait, passée une légitime interrogation quant à une telle amputation, la soirée ne sera que bonheur.

Très éloignée du lyrisme halluciné aux confins du fantastique de Die tote Stadt, Die Kathrin est une œuvre extrêmement touchante basée sur un livret plutôt classique dans son architecture. Comme chez Verdi, soprano et ténor voient leur amour contrarié par la basse, mais, et c'est là que l'œuvre atteint à l'universel, pas que : ce qui sépare surtout la très bonne personne qu'est la jeune Allemande Kathrin, de son adorable chanteur français François, c'est la guerre. À peine née, alors que, comme tout un chacun, dans tous les pays du Monde, Kathrin et François n'aspirent qu’à une vie simple (un amour, une maison), leur passion va devoir voyager. La Grande histoire qui s'invite dans la Petite, lot de tant d'êtres humains qui n'ont pas la chance d'être nés du bon côté de la frontière. Frontière des langues : Korngold fait malicieusement dialoguer le français et l'allemand dans une partition bilingue où l'on déclare sa flamme en V.O. et en V.F.: «Jet’aime/Ichliebe dich ». Le thème de la frontière ne va cesser d'irriguer l'opéra, surtout dans l'" adaptation dramaturgique et musicale » de Michel Pastore qui débaptise l’opéra de Korngold (Die Kathrin devient Kathrin vs Zone Libre), déconstruit l'ordonnance initiale en trois actes, remplacés ici par la succession des scènes voulues par le compositeur autrichien, et intitulées : François vs François, François vs Kathrin, Kathrin vs Zone libre, Guerre vs Guerre, Exil vs Kathrin, Exil vs François...

Ce versus, signifiant à côté ou en face de, dit tout des barrières qui vont s'élever entre les personnages au cours d'une œuvre véritablement prophétique, située en Allemagne, en France et même à.... Marseille : on y parle de la Canebière, on y fait sa « Tête de Voltaire », on se moque de la Jeanne d'Arc du Luna Park...), avant de tomber enfin pour un happy end campagnard sur fond $\bullet \bullet$ 
•• de couchant hollywoodien. Notons également le beau clin d'œil de l'allusion cinématographique au parcours hollywoodien de Korngold avec la mise en abîme du mélo de Douglas Sirk Le Mirage de la vie/L'amour d'une mère, film qui scelle la rencontre inaugurale de Kathrin et François. Oui, Die Kathrin est un mélo. D’une eau simplissime et pure. Qui va droit au cœur.

On connaît finalement assez mal l'Orchestre symphonique de la Garde républicaine. On découvre ce soir son excellence et son enthousiasme à jouer cette partition extrêmement lyrique, hors des sentiers qu'il emprunte habituellement, plutôt cantonné qu'il està la musique symphonique, aussi bien en France que lors de ses nombreuses tournées à l'étranger. Sébastien Billard dirige sa soixantaine de musiciens (oui, il y a même des cordes à la Garde républicaine!) avec précision, fougue, et une réelle empathie pour cette musique aux grandes envolées, riche en mélodies, mais également puissante en harmonies subtiles : on ne peut s'empêcher d'aller à la pêche aux influences : Puccini et sa Bohème pour les premiers échanges entre François et Kathrin ou le premier aria d'icelle... plus loin les quintes à vide de sa Barrière d'enfer, Strauss et sa Femme sans ombre sur « Je veux aller à lui » quasi décalque du « Ist mein liebster dahin? » de l'Impératrice, mais aussi la $5^{e}$ de Mahler dans certaine trompette des parties militaristes ou encore Chostakovitch, autre ultime romantique honni lui aussi en son temps par un autre totalitarisme.

La distribution rassemblée là est absolument exemplaire : Vincent Schirrmacher en François est sidérant d'insolence vocale, extrêmement généreux avec les décibels pucciniens requis. On lui souhaite de garder longtemps un tel outil qu'il lui faudra certainement ménager. Pour l'heure il est le révélateur le plus spectaculaire, véritablement toutes cordes dehors, de cette Kathrin inconnue. Sa partenaire est merveilleuse également : Elisabeth Flechl est très à l'aise avec l'exigeante partie du rôle-titre, qu'elle gratifie d'une ligne de chant au legato de miel, d'une longueur de souffle à toute épreuve, de sons filés enchanteurs : sa dernière tenue est celle d'une Isolde. Klemens Sander ne leur cède en rien et son méchant Malignac façon Scarpia (écouter son credo «Chasser, séduire, posséder »), est parfait d'articulation et de noblesse vocale. Ajoutons que sa ressemblance physique avec un certain Jonas Kaufmann sert à merveille son engagement scénique. Signalons que tous trois viennent du Volksoper de Vienne, où ils assurent en toute splendeur les nombreux rôles titres de la saison!
La soprano roumaine Liliana Faraon est une ChouChou ivre de sa voix. Son abattage en ferait une parfaite Musetta. Wilfried Tissot, familier du public de l'Opéra de Marseille, assume avec éclat les multiples interventions de petits rôles que Michel Pastore a rassemblés en un personnage unique appelé Le Témoin. Très jolies interventions parfaitement mesurées et timbrées, pour sa première apparition en scène, du jeune Alexandre Csoma dans le rôle de François, prénom très tendance dans cet opéra, puisque c'est celui de l'enfant que Kathrin a conçu avec... François.

Même amputé, Le Festival Musiques Interdites version 2014 a réussi son pari : cette Kathrin, amputée de même, mais bellement défrichée, et enregistrée ce mardi 8 juillet, donne envie de réécouter les Korngold que l'on connait et bien sûr d'aller plus loin, avec notamment une Kathrin complète sur scène. Cela viendra, n'en doutons pas. Son hérö̈ne ultra positive est de ces gens qui font honneur à l'humanité. Dans une époque plutôt dure, on aurait tort d'avoir le cœur sec, voire de s'agacer, face au discours de Kathrin : «Au cœur des ténèbres rayonne la lumière de l'espoir» «Aime l'adversité car te porte ta propre solitude. » Cette Kathrin n'a rien de « dégénéré », bien sûr.

Dans le beau film d'Olivier Ducastel et Jacques Martineau, L'arbre et la forêt, le héros, rescapé des camps, répondait à ceux qui s'étonnaient de sa passion intacte pour la musique de Wagner : «Les nazis m'ont tout pris. Ils ne vont pas en plus me prendre Wagner. »

On aura compris qu'en cette époque où la Mémoire donne çà et là d'inquiétants signes de vacillement, le Festival Musiques Interdites mérite hélas une place essentielle.

Vivent les Musiques Interdites, donc! Afin qu'il n’y en ait plus jamais.

Jean-Luc Clairet 